\title{
GOULD'S BELT
}

\author{
P. O. LINDBLAD \\ Stockholm Observatory, Sweden
}

The zone of bright stars inclined $20^{\circ}$ to the galactic plane, pointed out by John Herschel (1847) and by Gould $(1874,1879)$, is generally referred to as Gould's Belt. This General Assembly is well timed. With the splendour of the centre of the Milky Way in the zenith in the early hours of the night anyone who comes out of the city-lights of Sydney can try to convince himself of the reality of this phenomenon.

Shapley and Cannon $(1922,1924)$ showed that the concentration towards Gould's Belt is very pronounced for the brighter B stars, while the fainter ones are highly concentrated towards the galactic plane. Their results indicated the radius of this local system to be of the order of $500 \mathrm{pc}$. The motions of the B stars brighter than a magnitude of about $5^{m} \cdot 5$ are characterized by the K-effect, which thus seems to be a property of this local Gould Belt system.

Numerous investigations of the distribution and motion of the bright $B$ stars have been published. A very thorough analysis has been carried out by Mrs Lesh (1972b),

TABLE I

Velocity gradients as observed for nearby early type stars together with theoretical values for an expanding group and for pure differential rotation. Unit $1 \mathrm{~km} \mathrm{~s}^{-1} \mathrm{kpc}^{-1}$

\begin{tabular}{lllc}
\hline Gradient & Observed & $\begin{array}{l}\text { Exp. model } \\
\text { age } 60 \times 10^{6} \mathrm{yr}\end{array}$ & Diff. rot. \\
\hline$\frac{\delta U}{\delta X}$ & $+23 \pm 2$ & +26 & 0 \\
$\frac{\delta U}{\delta Y}$ & -16 & -8 & -25 \\
$\frac{\delta V}{\delta X}$ & +18 & -8 & -5 \\
$\frac{\delta V}{\delta Y}$ & +10 & +7 & 0 \\
\hline
\end{tabular}

who refined a method due to Blaauw and Bonneau. Table I shows the observed velocity gradient matrix as derived by Mrs Lesh (where $X$ and $U$ respectively represent position and velocity in the direction of the galactic anticentre, and $Y$ and $V$ corresponding quantities in the direction of galactic rotation). Assuming an expanding system in the gravitational field of the Galaxy, values for these gradients can be predicted for various expansion ages. The table gives these values for an expansion age of $60 \times 10^{6} \mathrm{yr}$, which seems to be close to a best fit. The table also gives expected gradients for pure differential rotation without expansion. $\delta U / \delta X$ and $\delta V / \delta Y$ agree 
well with the expanding model, while $\delta U / \delta Y$ is somewhat undecided and $\delta V / \delta X$ completely off for both models.

At the Stockholm Observatory we have been investigating the velocity-distribution of the very local neutral hydrogen as well as formaldehyde velocities of dark clouds along Gould's Belt (Lindblad et al., 1973). Dr Weaver will discuss the local gas this afternoon although he will give a slightly different interpretation. In Figure 1 the filled circles indicate velocities for a narrow $\mathrm{H}$ I component with very wide extent in galactic latitude. The open circles represent another, broader and more irregular, component with less extent in latitude, in some regions identical with what is called the Orion arm. These two components together constitute the local gas. Crosses represent formaldehyde observations, where crosses in boxes indicate clouds definitely associated with the inclined Gould's Belt.

It now proves that the velocity relation for the filled circles can be reproduced by the model of a slowly expanding cloud - or rather a doughnut - following the equations of motion given by Blaauw. The theoretical relation is shown by the full-drawn curve in Figure 1. The expansion age of the cloud as given by the best fit is $60 \times 10^{6} \mathrm{yr}$ and thus happens to coincide with the recent value given by Mrs Lesh. Figure 2 shows the position of the model dough-nut and the projection on the galactic plane of Mrs

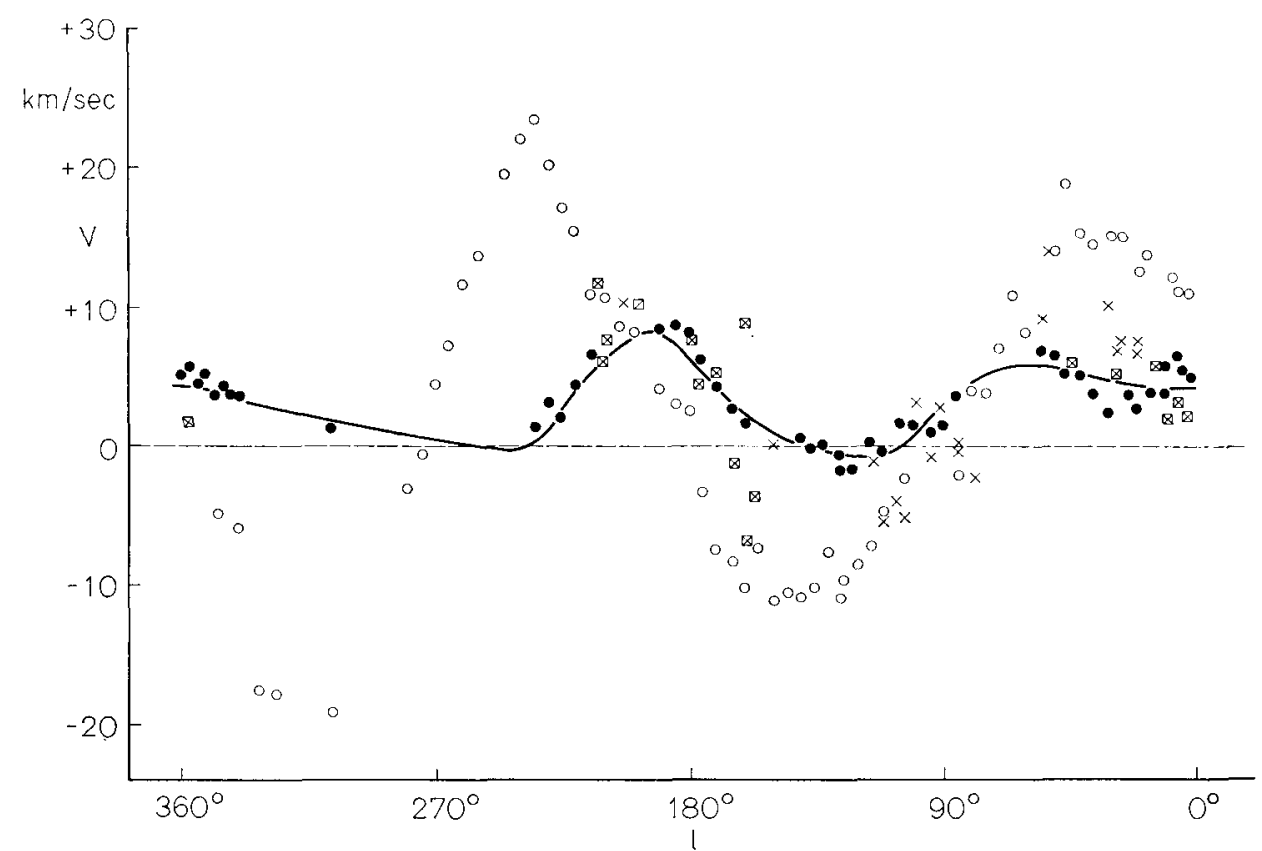

Fig. 1. Velocity-longitude diagram for the two components of local neutral hydrogen gas (filled and open circles). Crosses mark formaldehyde velocities, crosses within squares representing clouds falling in the Gould Belt region when separated from the galactic plane. The full-drawn line shows the theoretical relation for the expanding doughnut shaped cloud represented by the ellipse in Figure 2. (Figures 1 and 2 have been reproduced, with the kind permission of Springer Verlag, from: Proceedings of the First European Astronomical Meeting, Vol. 2, 'Stars and the Milky Way System', ed. L. N. Mavridis, Springer, Berlin-Heidelberg-New York, 1974.) 
Lesh's stars. The centre of the cloud falls in the neighbourhood of the Pleiades, and the original velocity of expansion is $3.6 \mathrm{~km} \mathrm{~s}^{-1}$.

The similarity of kinematic behaviour between the neutral hydrogen, the dark clouds along Gould's Belt and the Gould Belt stars, would indicate that this local hydrogen component is related to the expanding Gould Belt system, and that this system was very much more concentrated $60 \times 10^{6} \mathrm{yr}$ ago. One may then speculate that the original compression occured in a spiral shock. The expansion age and a knowledge of the present position of the shock then could give a valuable check on the spiral pattern velocity.

However, once we introduce the density wave theory we are led to examine carefully if this gas cloud and local system really exists as such, as expansion is a general phenomenon in inter-shock regions. The effect to consider is velocity-crowding and can be exemplified by an investigation by Roberts (1972). In Figure 3 of Roberts' paper we see how in a spiral shockwave pattern the gas density and velocity may vary along the line of sight at a certain longitude. This gives rise to a spurious component

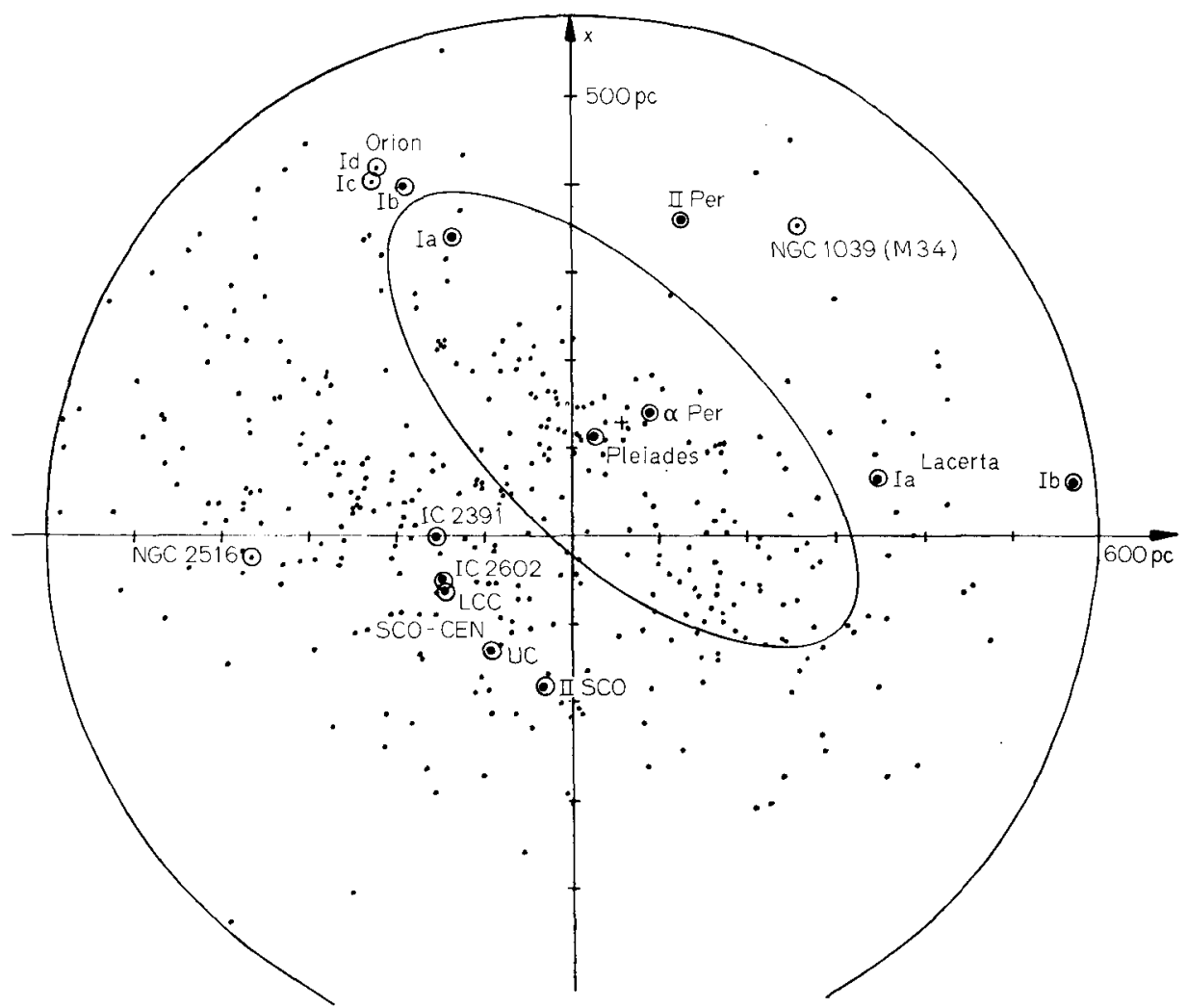

Fig. 2. Projections on the galactic plane of stars earlier than B5 with well determined distances smaller than $600 \mathrm{pc}$ according to Lesh $(1968,1972 \mathrm{a})$. The centre of the coordinate system marks the position of the Sun and the positive $X$-axis points towards the galactic anticentre. The ellipse with its centre marked by a cross represents the ring model for the neutral hydrogen described in the text. 
close to zero velocity in the resulting line-profile, due to the tenuous inter-arm gas between us and the shock. Could the hydrogen component dealt with here be due to velocity crowding in an otherwise featureless inter-arm gas? This possibility is at present being investigated in Stockholm applying Roberts' non-linear shock wave theory. Burton, who presently is applying the linear density wave theory on this problem, reports positive results (see the discussion below).

It is then of great importance to establish whether the stars form a separate local system or not. The observations we would like to suggest may be illustrated by a figure due to Dixon (1970). Dixon plotted radial velocities vs distance for stars of two age-groups in the direction towards the galactic centre and anticentre in order to determine $\delta U / \delta X$. However, when examining Dixon's figure it is interesting to note that the velocity gradients would be $26 \mathrm{~km} \mathrm{~s}^{-1} \mathrm{kpc}^{-1}$ for an expanding group of age $60 \times 10^{6} \mathrm{yr}$ and would be 0 for pure differential rotation. Dixon's diagram may suggest a high velocity gradient in the solar neighbourhood which shifts back to zero at larger distances indicating a local expanding system. First order density wave theory, on the other hand, would give a sine-wave with minima at the density maxima of the spiral arms.

If we could establish, at longitudes where the gradients of different models differ greatly, a clear discontinuity in the radial velocities as a function of distance from the Sun, we could determine the extent of the expanding Gould Belt system in different directions and perhaps determine an upper limit for the age of its stellar population.

\section{References}

Dixon, M. E.: 1970, Monthly Notices Roy. Astron. Soc. 151, 87.

Gould, B. A.: 1874, Proc. Am. Assoc. Adv. Sci. 1874, 115.

Gould, B. A.: 1879, Uranometria Argentina. Result. Obs. Nac. Argentina Cordoba 1, 355.

Herschel, J. F. W.: 1847, Results of Astronomical Observations Made During the Years 1834, 5, $6,7,8$, at the Cape of Good Hope, Smith, Elder and Co., London, p. 385.

Hobbs, L. M.: 1971, Astrophys. J. 166, 333.

Lesh, J. R.: 1968, Astrophys. J. Suppl. 17, 371.

Lesh, J. R.: 1972a, Astron. Astrophys. Suppl. 5, 129.

Lesh, J. R.: 1972b, in G. Cayrel de Strobel and A. M. Delplace (eds.), 'L'âge des étoiles', IAU Colloq. 17, Paper XXIII.

Lindblad, P. O., Grape, K., Sandqvist, Aa., and Schober, J.: 1973, Astron. Astrophys. 24, 309.

Roberts, W. W.: 1972, Astrophys. J. 173, 259.

Shapley, H. and Cannon, A. J.: 1922, Harvard Circ. 239.

Shapley, H. and Cannon, A. J.: 1924, Harvard Repr. 6.

\section{DISCUSSION}

Burton: There is evidence on a large scale that the motions of the neutral hydrogen in the Galaxy are ordered according to the general predictions of the linear density-wave theory. It is reasonable to examine the effects of those motions on a small scale (say, less than $0.5 \mathrm{kpc}$ ) near the Sun. T. M. Bania and I at NRAO are doing this; our results show that most of the observational characteristics of Lindblad's feature A can be accounted for by the sort of flow pattern observed on a large scale also being present near the Sun. This explanation of course does not require special local mechanisms. Specifically, we found the flow parameters required by the hydrogen-line profile cut-offs which are contributed locally (positive cut-off at $70^{\circ}<l<170^{\circ}$, negative velocity cut-off at $190^{\circ}<l<290^{\circ}$ ). 
These cut-offs contain unambiguous velocity information and are easier to isolate than features deeper in the profiles. The derived flow parameters are consistent with those found to hold on a large scale. When model hydrogen profiles are calculated using these parameters, a feature results which shows approximately the same velocity-longitude relationship as found by Lindblad for feature A. Our preliminary results suggest that some of the kinematic characteristics (projected to $b=0^{\circ}$ ) of the Gould Belt stars can also be accounted for by this simple model.

Lindblad: This is the investigation I was referring to. I think the results are very interesting.

Toomre: Does either Dr Lindblad or Dr Burton have any theoretical idea why that Belt should be tilted?

Lindblad and Burton: We have no idea.

Buscombe: The high-resolution optical interstellar gas velocities, e.g. by Hobbs, should indicate which components are caused closer to the Sun than the stars. How do they fit with the H I observations you have mentioned?

Lindblad: One interesting observation which has to be explained is that Hobbs' (1971) interstellar lines in the spectra of the Pleiades stars already show the positive velocities which our model would ascribe to gas further away. 